\title{
La Medición de la Eficiencia Universitaria: Una Aplicación del Análisis Envolvente de Datos
}

\author{
Raquel Martín \\ Universidad de La Laguna, Facultad de Ciencias Económicas y Empresariales, Departamento \\ de Economía de las Instituciones, Estadística Económica y Econometría, Cno. La Hornera s/n, \\ Campus de Guajara, 38071 La Laguna, Tenerife, Islas Canarias-España (e-mail: rmartinr@ull.es)
}

\section{Resumen}

En este artículo se presenta un análisis del grado de eficiencia técnica con que actúan los departamentos universitarios. En este caso la universidad elegida ha sido la Universidad de La Laguna, llevando a cabo el análisis para un curso académico. La metodología utilizada es el Análisis Envolvente de Datos, análisis que permite conocer mejor como funcionan los departamentos universitarios. Además, la metodología suministra información para mejorar la eficiencia y facilitar la gestión universitaria. La principal conclusión del estudio es que la asignación de los recursos destinados a la educación superior, es susceptible de ser mejorada en los aspectos concernientes a la eficiencia en el uso de los mismos.

Palabras clave: eficiencia universitaria, educación superior, gestión, análisis envolvente de datos

\section{Measuring University Efficiency: An Application of Data Envelopment Analysis}

\begin{abstract}
The degree of technical efficiency with which university departments develop their activities is analyzed. As study case the analysis is done using data for one academic year in the University of La Laguna in Spain. The methodology used is the Data Envolvent Analysis, method that allows knowing better the functioning of the university departments. In addition, the methodology gives information to improve efficiency and to facilitate the university management. The most important conclusion of the study is that the resources assigned to higher education can be improved in aspects related to the efficiency in the use of them.
\end{abstract}

Keywords: university efficiency, higher education, management, data envelopment analysis 


\section{INTRODUCCIÓN}

Las universidades públicas de los países desarrollados han experimentado transformaciones importantes derivadas, principalmente, de los cambios producidos en su contexto. Dentro de estos cambios, las presiones financieras sobre el Sector Público, concretadas, por ejemplo, en la búsqueda de la reducción del déficit, han producido una creciente preocupación por la medida de la eficiencia con la que desarrollan sus actividades las distintas organizaciones que forman dicho sector (Lovell y Muñiz, 2003). A su vez, otros aspectos han generado un incremento de los estudios referentes a la eficiencia y a su evaluación en el ámbito del Sector Público, como son el aumento de las exigencias por parte de los usuarios para obtener mayores niveles de eficiencia y efectividad de los servicios públicos consumidos o la gran inquietud social por dotar a los centros de decisión de las entidades públicas de modernas técnicas de gestión que contribuyan eficazmente al proceso de toma de decisiones (Yetano, 2007). En consecuencia, el interés por la medida y la mejora de la eficiencia con la que actúan las instituciones encargadas de impartir educación superior universitaria ha ido cobrando una importancia bastante significativa.

En el caso de las universidades públicas, dado que se trata de organizaciones que gestionan sus recursos con considerable autonomía, la producción universitaria ha sido objeto de especial atención por parte de la literatura económica. Además, Europa se encuentra en el proceso de creación del "Espacio Europeo de Educación Superior", que supone un fuerte incremento de la competencia entre instituciones universitarias y la búsqueda de situaciones caracterizadas por la eficiencia y la calidad de los servicios ofrecidos, derivado del entorno competitivo. Paralelamente, se está ante un escenario de cambio de la legislación universitaria española, que introduce un órgano de evaluación de los resultados universitarios, la Agencia Nacional de Evaluación y Acreditación (ANECA), que ha supuesto que los procesos de evaluación estén de plena actualidad.

La hipótesis de partida en esta investigación ha sido que la asignación de los recursos destinados a la educación superior, es susceptible de ser mejorada en los aspectos concernientes a la eficiencia en el uso de los mismos. Situados en este contexto, la presente investigación se ha centrado en la determinación de la eficiencia con la que actúan las instituciones universitarias, como paso previo para la mejora en la asignación de recursos, y por tanto, de la gestión. En el caso de la universidad elegida (Universidad de La Laguna, ULL), se han tomando como unidades de análisis sus departamentos. Los trabajos de este tipo son relativamente recientes en España y en Canarias, concretamente, no existen antecedentes empíricos.

Existen diversas técnicas que ayudan a enfocar económicamente los problemas de gestión pública (Álvarez, 2001). Pero las peculiaridades de la producción de servicios en el Sector Público convierten unas técnicas en más idóneas que otras. Dentro de las diferentes metodologías existentes, el Análisis Envolvente de Datos (DEA) (Coelli et al., 1999; Charnes et al., 1994) se ha convertido en una técnica usualmente empleada para evaluar la eficiencia, con la que actúan unidades tomadoras de decisiones, como es en este caso los departamentos universitarios. El análisis de la eficiencia técnica con la que actúan los departamentos universitarios, a través de la metodología DEA, permitirá profundizar en el conocimiento de la tecnología de producción de los departamentos universitarios y suministrará información para mejorar la eficiencia y, por tanto, facilitar la gestión universitaria. En este contexto, el objetivo de este trabajo es analizar la eficiencia técnica en los departamentos de la Universidad de La Laguna (ULL).

\section{METODOLOGÍA Y DATOS}

En la aplicación del DEA que se realiza en esta investigación, se utilizan como "unidades tomadoras de decisiones" los departamentos universitarios de la ULL, efectuando un análisis de la eficiencia con que desarrollan sus funciones docentes e investigadoras (outputs), a partir de los recursos materiales, humanos y financieros (inputs) de los que disponen, puesto que, tal y como recoge la LOU (Ley Orgánica 4/2007, de 12 de abril, por la que se modifica la Ley Orgánica 6/2001, de 21 de diciembre, de Universidades), en su artículo 9, los departamentos son las unidades de docencia e investigación encargadas de coordinar las enseñanzas de uno o varios 
ámbitos del conocimiento en uno o varios centros, de acuerdo con la programación docente de la universidad, de apoyar las actividades e iniciativas docentes e investigadoras del profesorado.

Además de lo anterior, también se debe tener en cuenta que las actividades de docencia e investigación son producidas a partir de un conjunto de inputs comunes a ambas: los profesores y recursos económicos y materiales. Es por ello que se considera que las actividades de docencia e investigación son actividades interrelacionadas y que, por tanto, deben ser analizadas conjuntamente. Asimismo, en las universidades también pueden aparecer sinergias como resultado de la producción conjunta de docencia e investigación. Tal como señala Martínez (2003), la investigación puede incidir positivamente en la calidad docente, la especialización del tercer ciclo, el diseño del currículo o la provisión de equipos e infraestructuras a emplear en actividades docentes. Los datos utilizados en este análisis empírico hacen referencia al curso académico 2002/2003 y proceden de la Memorias Académicas de la ULL, de las Memorias de Investigación, de las Guías de la Comisión de Doctorado, del Informe de Evaluación de la Calidad Docente, de los presupuestos de la Universidad, de la información suministrada por el Gabinete de Análisis y Planificación (GAP), por el Vicerrectorado de Investigación, por la Unidad Técnica de Evaluación (UTE) y por la Oficina de Transferencia de Resultados de Investigación (OTRI) de la ULL.

La identificación de las variables que representan apropiadamente la actividad productiva de las organizaciones resulta fundamental para aproximarnos a la eficiencia real de las entidades evaluadas, sin embargo, a la hora de aplicar el DEA, se presentan dificultades en la definición, selección y medida de las variables (Martín, 2007). Los argumentos de tipo teórico que suministra la Economía de la Educación, junto a la revisión de la literatura sobre trabajos previos, que han estimado una función de producción en las instituciones de educación superior y la disponibilidad de información, son los que fundamentan las variables seleccionadas en este estudio. En cuanto al número de variables finalmente utilizado, se ha tenido en cuenta que su cuantía afecta a la capacidad de discriminación del DEA, en el sentido de que, cuanto mayor es el número de variables con relación al número de unidades analizadas, mayor será la probabilidad que tiene cada una de ellas de presentar un buen comportamiento en alguna de las variables consideradas y por tanto, de conseguir un índice de eficiencia unitario (Nunamaker, 1985).

En cuanto a los outputs, que definen la función de producción, se diferencian aquéllos que hacen referencia a la docencia y aquéllos que se refieren a la investigación. En relación a los primeros, se han utilizado como variables cuantitativas las siguientes: el $n^{\circ}$ de créditos impartidos por departamento (NC), el $n^{\circ}$ de alumnos matriculados en primer y segundo ciclo por departamento (NA), y el $n^{\circ}$ de alumnos de tercer ciclo por departamento (NATC). Como variable cualitativa se ha utilizado el porcentaje de profesores que han obtenido una buena valoración en la encuesta de evaluación docente por departamento (PED) (con puntuación igual o superior a 4, en una escala de 1 a 5), donde se valoran diferentes aspectos (dominio, conocimiento didáctico, organización, motivación, medios y satisfacción). Con respecto a la investigación, se han utilizado como variables el número de publicaciones (NP) y los ingresos por proyectos de investigación (IPI). Siguiendo algunos trabajos que analizan la investigación universitaria (Martínez, 2003; Ng y Li, 2000) se utiliza el valor acumulado de la producción científica para varios cursos académicos: $2002 / 2003$ y 2001/2002. Con respecto al "número de publicaciones" (NP), se ha incluido el número de publicaciones nacionales e internacionales con una ponderación de 1 , y el número de ponencias y comunicaciones a congresos con una ponderación de 0,6. En relación a los ingresos de proyectos de financiación, éstos eran principalmente de 3 categorías: 1 año, 2 años y 3 años, y para imputarlos al período considerado se ha hecho un reparto proporcional. Lamentablemente, no se ha podido incluir ningún tipo de ponderación que permita considerar la calidad de la producción científica.

En relación a los inputs que caracterizan la tecnología de producción, éstos se clasifican en recursos humanos, financieros y materiales. Con respecto a los recursos humanos se han agrupado a los profesores en dos tipos: los de perfil "a priori" mixto (PDI), dentro de los cuales se ha considerado a los catedráticos de universidad, a los catedráticos de escuela y a los titulares de universidad; y los profesores que por la naturaleza jurídica de sus puestos están especializados 
en labores de docencia (PD), formados por los titulares de escuela, los profesores asociados y ayudantes. En ambos grupos existe personal con una dedicación parcial (a 3, 4, 5 y 6 horas). Éstos han sido ponderados con $0,375,0,5,0,625$ y 0,75 respectivamente, obteniendo como resultado el profesorado existente equivalente a tiempo completo. Con respecto a los recursos financieros se ha utilizado como variable el presupuesto departamental (PP). Éste es anual, mientras que el análisis hace referencia a un curso académico. Por ello, se consideraron los presupuestos de los años 2003 y 2002, prorrateando las cantidades y calculando la parte proporcional correspondiente.

Tabla 1: Variables de la función de producción.

\begin{tabular}{|l|l|}
\hline \multicolumn{1}{|c|}{ Inputs } & \multicolumn{1}{c|}{ Outputs } \\
\hline Recursos Humanos: & Docencia: \\
- Catedráticos universidad, catedráticos & $-\%$ profesores buena valoración encuesta (PED). \\
escuela y titulares universidad (PDI). & $-\mathrm{N}^{\circ}$ créditos impartidos por departamento (NC). \\
- Titulares escuela, profesores & $-\mathrm{N}^{\circ}$ alumnos matriculados en $1^{\text {er }}$ y $2^{\circ}$ ciclo (NA). \\
asociados y ayudantes (PD). & $-\mathrm{N}^{\circ}$ alumnos de $3^{\text {er }}$ ciclo por departamento (NATC). \\
\hline Recursos Financieros: & - Investigación: \\
- Presupuesto departamental (PP). & $-\mathrm{N}^{\circ}$ de publicaciones (NP). \\
& - Ingresos por proyectos de investigación (IPI). \\
\hline
\end{tabular}

En cuanto a la metodología utilizada, el Análisis Envolvente de Datos, desarrollado por Charnes et al. (1978), a partir del trabajo seminal de Farrell (1957), tiene por objeto la medición de la eficiencia de lo que sus autores denominaron "unidades tomadoras de decisiones" (DMU), unidades que se deben caracterizar por consumir el mismo tipo de inputs para la obtención del mismo tipo de outputs, esto es, deben ser unidades homogéneas. EI DEA trata de definir la frontera de producción empírica formada por las mejores unidades observadas, para posteriormente cuantificar el grado de eficiencia de las observaciones que forman parte de la muestra, es decir, su distancia en relación con la frontera. Así, el DEA compara a cada unidad únicamente con las mejores unidades observadas, siendo el indicador de eficiencia que se obtiene relativo.

Tabla 2: Formulación básica del DEA: modelo CCR.

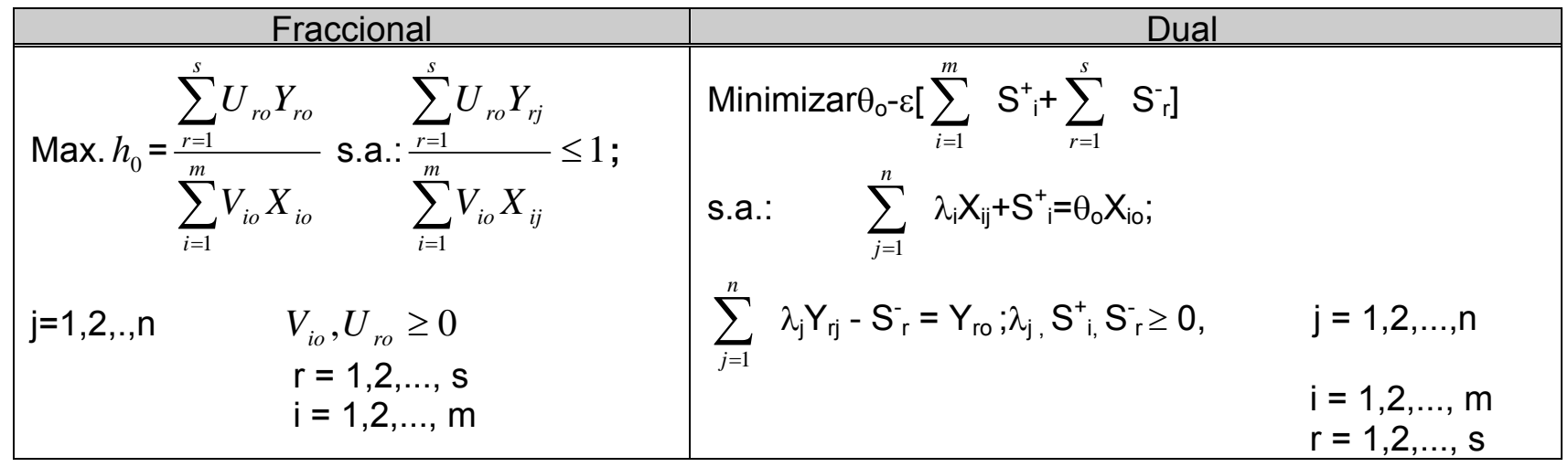

Para medir la eficiencia, cuando nos encontramos con múltiples inputs y outputs, surge la formulación propuesta por Charnes et al. (1978), conocida como modelo CCR, que originariamente adopta la forma fraccional expuesta en la tabla 2. El indicador de eficiencia se define como el cociente entre la suma ponderada de los outputs y la suma ponderada de los inputs, ponderaciones que son generadas por la propia técnica a través de la programación matemática, obteniendo, para cada unidad, la valoración más favorable de su eficiencia en relación con las demás. El valor del índice de eficiencia oscila entre cero y uno. Las unidades que obtienen el valor unitario son aquéllas que son eficientes, mientras que la obtención de un valor inferior implica la existencia de ineficiencia. La complejidad que presenta la resolución del programa en forma fraccional llevó a Charnes et al. (1979) a transformarlo en uno equivalente de programación lineal. La versión utilizada en la mayoría de los trabajos aplicados es el dual del programa lineal, puesto que cada una de las variables en él tiene un significado concreto y es una formulación más sencilla (Ganley y Cubbin, 1992). 
El modelo expuesto hasta ahora contiene la restricción de rendimientos constantes a escala, lo que implica que es posible que unidades de gran escala sean comparadas con unidades hipotéticas de reducida escala y al contrario, hecho que en la práctica puede ser inapropiado. Para detectar las posibles fuentes de ineficiencia que provienen de la escala de operaciones en la que opera una DMU, surge el modelo BCC planteado por Banker et al. (1984). En cuanto a la elección del modelo DEA, entre un modelo DEA con rendimientos constantes a escala (CCR) y uno con rendimientos variables de escala (BCC), se han utilizado los procedimientos descritos por Martínez (2003), y que han sido utilizados posteriormente en otros trabajos como en el realizado por Calderón et al. (2005). Dichos procedimientos han sido: Regresión de los índices de eficiencia CCR sobre el tamaño de los departamentos (medido por el número de profesores equivalentes a tiempo completo), lo que permite comprobar si el tamaño es estadísticamente significativo para explicar los índices de eficiencia; contraste de Mann-Whitney (para contrastar la hipótesis de que los dos grupos independientes de los índices de eficiencia proceden de la misma población) y contraste de Kolmogorov-Smirnov (para contrastar la hipótesis de que la distribución de los índices de eficiencia es la misma) sobre los índices de eficiencia calculados para los modelos CCR y BCC; coeficiente de correlación entre los índices de eficiencia obtenidos para los dos modelos: CCR y BCC (un coeficiente de correlación alto indica que la aplicación de las dos formulaciones del modelo proporcionan como resultado ordenaciones muy parecidas); y eficiencia de escala (que se obtiene a través del cociente entre los índices de eficiencia obtenidos por el modelo CCR y los índices de eficiencia obtenidos por el modelo BCC). Los procedimientos utilizados permiten señalar que la tecnología de producción, que presentan los departamentos de la ULL, para el curso académico 2002/2003, y con los datos considerados, se caracteriza por mostrar rendimientos constantes a escala. Es por ello que se ha utilizado el modelo CCR.

En relación a la orientación del modelo, se considera que el sector universitario se caracteriza porque el grado de control de los gestores de las unidades objeto de análisis sobre sus recursos es mínimo, ya que éstos vienen determinados por niveles superiores de la administración en función de unos criterios preestablecidos y fundamentalmente basados en la demanda. Por tanto, parece razonable suponer que los objetivos de los gestores públicos irán orientados hacia la obtención de los mejores resultados, en lugar de hacia una minimización de los recursos (Martínez, 2003; Avkiran, 2001; Caballero et al., 2000). Ello lleva a seleccionar un modelo DEA con orientación al output para la evaluación de la eficiencia, es decir, se analizará en qué porcentaje los departamentos universitarios pueden incrementar su producción docente e investigadora, a partir de los recursos disponibles.

Por otra parte, la evaluación de la eficiencia a través de la metodología DEA exige que las unidades productivas, objeto de análisis, sean homogéneas. En los trabajos que aplican el DEA sobre el conjunto de actividades de los departamentos de una misma universidad (Johnes, 2006; Caballero et al., 2000, 2004; Giménez y Martínez, 2001; García, 1996; Sinuany-Stern et al., 1994), se encuentran unidades muy diversas que pueden dar cierto grado de heterogeneidad. Para dar homogeneidad a las unidades a analizar, en las investigaciones centradas en la educación universitaria, los trabajos analizados diferencian los departamentos agrupándolos según el grado de experimentalidad. En el caso de este estudio, se ha considerado la diferenciación de los departamentos según sean experimentales o no, realizando un análisis DEA para cada uno de los grupos por separado. Por último, para el caso de la ULL, las ventajas y las restricciones del DEA son las resumidas en la Tabla 3.

Tabla 3: Ventajas y restricciones del DEA.

\begin{tabular}{|l|l|}
\hline \multicolumn{1}{|c|}{ Ventajas } & \multicolumn{1}{c|}{ Restricciones } \\
\hline -Se adapta a múltiples inputs y outputs. & -Incapacidad de identificar outliers. \\
-No contempla la ausencia de precios. & -Modelo determinístico. \\
-Evita la imposición de una forma funcional. & -Homogeneidad en las unidades analizadas. \\
-Ofrece información minuciosa y detallada. & -Necesidad proporción óptima de variables. \\
-Permite incluir factores fuera del control de DMU. & -Incapacidad de ordenar unidades eficientes. \\
-Permite variables expresadas distintas medidas. & -Resultados sensibles a la especificación. \\
-Flexibilidad. & -Flexibilidad. \\
\hline
\end{tabular}




\section{APLICACIÓN DEL DEA: RESULTADOS Y DISCUSIÓN}

El número total de departamentos en la ULL para el curso académico 2002/2003 era de 62, de los cuales 31 eran departamentos experimentales ( 8 correspondientes a la división de ciencias de la salud y 23 a la de ciencias experimentales y técnicas) y 31 eran no experimentales (21 correspondientes a la división de ciencias sociales, económicas, jurídicas y de la educación y los 10 restantes pertenecientes a humanidades). Existen departamentos que obtienen en alguna de las dos variables referidas al profesorado un valor nulo. Un modelo CCR con una orientación output sin inputs carece de sentido, por lo que finalmente se han eliminado los departamentos con algún input cero. Asimismo, el departamento 4 ha sido excluido por sus características particulares. Como consecuencia, el análisis ha sido realizado para un conjunto de 57 departamentos, de los cuales 28 son experimentales y 29 son no experimentales.

A continuación se recogen los índices de eficiencia obtenidos para los departamentos experimentales (tabla 4) y para los no experimentales (tabla 5), obtenidos a través del programa informático EMS (Efficiency Measurement System) de Holger Scheel (University of Dortmund).

En relación a los primeros, los resultados muestran 13 unidades eficientes y 15 ineficientes, siendo la media de las ratios de eficiencia del $91,9 \%$, lo cual supone un $8,1 \%$ de ineficiencia media para el conjunto de departamentos analizados. Adicionalmente se observa que los departamentos menos eficientes son los departamentos $35,47,10$ y 57.

Tabla 4: Índices de eficiencia. Departamentos experimentales.

\begin{tabular}{|l|c|c|c|c|c|}
\hline \multicolumn{1}{|c|}{ Departamento } & $\begin{array}{c}\text { Índices } \\
\text { eficiencia }\end{array}$ & Departamento & $\begin{array}{c}\text { Índices } \\
\text { eficiencia }\end{array}$ & Departamento & $\begin{array}{c}\text { Índices } \\
\text { eficiencia }\end{array}$ \\
\hline \hline Departamento 2 & 0,99 & Departamento 33 & 0,90 & Departamento 48 & 1 \\
\hline Departamento 3 & 1 & Departamento 34 & 1 & Departamento 49 & 1 \\
\hline Departamento 5 & 0,91 & Departamento 35 & 0,65 & Departamento 50 & 1 \\
\hline Departamento 6 & 0,88 & Departamento 36 & 1 & Departamento 51 & 0,86 \\
\hline Departamento 9 & 1 & Departamento 42 & 1 & Departamento 52 & 1 \\
\hline Departamento 10 & 0,72 & Departamento 43 & 1 & Departamento 57 & 0,73 \\
\hline Departamento 24 & 0,86 & Departamento 44 & 0,98 & Departamento 58 & 0,79 \\
\hline Departamento 25 & 1 & Departamento 45 & 1 & Departamento 59 & 0,97 \\
\hline Departamento 26 & 0,91 & Departamento 46 & 0,87 & & \\
\hline Departamento 27 & 1 & Departamento 47 & 0,71 & & \\
\hline
\end{tabular}

Con respecto a los departamentos no experimentales, se identifican 17 unidades eficientes y 12 ineficientes, siendo la media de los ratios de eficiencia de $93,59 \%$, lo cual indica un $6,41 \%$ de ineficiencia media para el conjunto de departamentos analizados. Dentro de ellos, los departamentos menos eficientes son los departamentos 14,53 y 31 .

Tabla 5: Índices de eficiencia. Departamentos no experimentales.

\begin{tabular}{|l|c|c|c|c|c|}
\hline \multicolumn{1}{|c|}{ Departamento } & $\begin{array}{c}\text { Índices } \\
\text { eficiencia }\end{array}$ & Departamento & $\begin{array}{c}\text { Índices } \\
\text { eficiencia }\end{array}$ & Departamento & $\begin{array}{c}\text { Índices } \\
\text { eficiencia }\end{array}$ \\
\hline \hline Departamento 1 & 0,825 & Departamento 20 & 0,948 & Departamento 38 & 1 \\
\hline Departamento 8 & 1 & Departamento 21 & 0,904 & Departamento 39 & 0,962 \\
\hline Departamento 11 & 1 & Departamento 22 & 1 & Departamento 40 & 1 \\
\hline Departamento 12 & 1 & Departamento 23 & 0,955 & Departamento 42 & 1 \\
\hline Departamento 14 & 0,64 & Departamento 28 & 1 & Departamento 53 & 0,678 \\
\hline Departamento 15 & 0,848 & Departamento 29 & 0,924 & Departamento 54 & 1 \\
\hline Departamento 16 & 1 & Departamento 30 & 1 & Departamento 55 & 1 \\
\hline Departamento 17 & 1 & Departamento 31 & 0,736 & Departamento 56 & 1 \\
\hline Departamento 18 & 1 & Departamento 32 & 0,862 & Departamento 61 & 1 \\
\hline Departamento 19 & 0,859 & Departamento 37 & 1 & & \\
\hline
\end{tabular}


Asimismo el DEA nos suministra información acerca de los grupos de referencia de cada unidad, los cuales vienen definidos por aquellos subconjuntos de entidades eficientes, que matemáticamente están determinados por aquellas unidades con valores de $\lambda_{\mathrm{j}}$ no nulos al resolver el problema de programación lineal. El conocimiento de la composición del grupo de referencia de cada uno de los departamentos ineficientes puede resultar inestimable, a la hora de implementar estrategias encaminadas a mejorar la eficiencia. En este sentido, las prácticas productivas de cada entidad ineficiente deben compararse con aquéllas que forman parte del grupo de referencia.

Otra información adicional, que puede obtenerse a partir del grupo de referencia, es la importancia relativa que ha tenido cada uno de los componentes del grupo en la determinación del departamento ficticio con el que ha sido comparada la actividad de cada uno de los departamentos ineficientes. Esta información viene suministrada por los valores de las ponderaciones $\lambda_{\mathrm{j}}$ asignadas por el modelo a cada uno de los departamentos que forman parte del grupo de referencia. El objetivo principal es poder llegar a conocer cuáles son los modelos de gestión a seguir por cada departamento ineficiente, puesto que cuanto mayor es el valor de los $\lambda_{\mathrm{j}}$, más parecido es el modelo de producción del departamento ineficiente al eficiente que forma parte del grupo de comparación. Por tanto, cada departamento, a la hora de adoptar medidas correctoras encaminadas a alcanzar una situación eficiente debe prestar especial atención a las prácticas productivas que se desarrollan en los departamentos que forman parte de su grupo de comparación.

Tabla 6: Grupos de referencia y ponderaciones $\lambda_{\mathrm{j}}$ asignadas. Departamentos experimentales.

\begin{tabular}{|c|l|l|}
\hline Departamento & Grupo de referencia & \multicolumn{1}{|c|}{ Ponderaciones } \\
\hline \hline 2 & 9,45 y 52 & $\lambda_{9}=0,957 ; \lambda_{45}=0,209 ; \lambda_{52}=0,819$ \\
\hline 5 & $3,9,34,36,48$ y 49 & $\lambda_{3}=0,23 ; \lambda_{9}=0,05 ; \lambda_{34}=0,19 ; \lambda_{36}=0,88 ; \lambda_{48}=0,004 ; \lambda_{49}=0,11$ \\
\hline 6 & $3,9,36,49$ y 52 & $\lambda_{3}=0,685 ; \lambda_{9}=0,294 ; \lambda_{3} 6=0,901 ; \lambda_{49}=0,080 ; \lambda_{52}=0,054$ \\
\hline 10 & $36,43,48$ y 52 & $\lambda_{36}=0,046 ; \lambda_{43}=0,620 ; \lambda_{48}=0,444 ; \lambda_{52}=0,149$ \\
\hline 24 & $3,9,36$ y 52 & $\lambda_{3}=0,027 ; \lambda_{9}=0,373 ; \lambda_{36}=0,699 ; \lambda_{52}=0,384$ \\
\hline 26 & $9,36,45,48$ y 52 & $\lambda_{9}=1,113 ; \lambda_{36}=0,663 ; \lambda_{45}=0,402 ; \lambda_{48}=0,027 ; \lambda_{52}=0,244$ \\
\hline 33 & $9,45,48$ y 52 & $\lambda_{9}=0,611 ; \lambda_{45}=0,477 ; \lambda_{48}=0,044 ; \lambda_{52}=0,151$ \\
\hline 35 & $9,42,45$ y 52 & $\lambda_{9}=1,798 ; \lambda_{42}=0,036 ; \lambda_{45}=1,048 ; \lambda_{52}=0,520$ \\
\hline 44 & 3,9 y 36 & $\lambda_{3}=0,496 ; \lambda_{9}=1,033 ; \lambda_{36}=0,551$ \\
\hline 46 & $9,36,43,48$ y 52 & $\lambda_{9}=0,041 ; \lambda_{36}=0,022 ; \lambda_{43}=0,859 ; \lambda_{48}=0,113 ; \lambda_{52}=0,021$ \\
\hline 47 & 48 y 52 & $\lambda_{48}=0,136 ; \lambda_{52}=0,967$ \\
\hline 51 & $36,43,45,48$ y 52 & $\lambda_{36}=0,261 ; \lambda_{43}=0,522 ; \lambda_{45}=0,083 ; \lambda_{48}=0,012 ; \lambda_{52}=0,178$ \\
\hline 57 & $3,9,36,43$ y 49 & $\lambda_{3}=0,326 ; \lambda_{9}=0,315 ; \lambda_{36}=0,187 ; \lambda_{43}=0,054 ; \lambda_{49}=0,377$ \\
\hline 58 & 3,9 y 36 & $\lambda_{3}=0,863 ; \lambda_{9}=0,057 ; \lambda_{36}=0,149$ \\
\hline 59 & $3,9,36,43$ y 49 & $\lambda_{3}=0,005 ; \lambda_{9}=0,114 ; \lambda_{36}=0,703 ; \lambda_{43}=0,202 ; \lambda_{49}=0,213$ \\
\hline
\end{tabular}

Tabla 7: Grupos de referencia y ponderaciones $\lambda_{\mathrm{j}}$ asignadas. Departamentos no experimentales.

\begin{tabular}{|c|l|l|}
\hline Departamento & Grupo de referencia & \multicolumn{1}{c|}{ Ponderaciones } \\
\hline \hline 1 & $16,22,30,40$ y 55 & $\lambda_{16}=0,089 ; \lambda_{22}=0,243 ; \lambda_{30}=0,036 ; \lambda_{40}=0,176 ; \lambda_{55}=0,219$ \\
\hline 14 & $8,22,40$ y 55 & $\lambda_{8}=0,016 ; \lambda_{22}=0,211 ; \lambda_{40}=0,938 ; \lambda_{55}=0,192$ \\
\hline 15 & $8,28,30$ y 55 & $\lambda_{8}=1,409 ; \lambda_{28}=0,521 ; \lambda_{30}=0,052 ; \lambda_{55}=0,046$ \\
\hline 19 & $8,40,55$ y 56 & $\lambda_{8}=0,672 ; \lambda_{40}=1,143 ; \lambda_{55}=0,099 ; \lambda_{56}=0,237$ \\
\hline 20 & 8,40 y 55 & $\lambda_{8}=0,359 ; \lambda_{40}=0,481 ; \lambda_{55}=0,397$ \\
\hline 21 & $8,22,40$ y 55 & $\lambda_{8}=0,624 ; \lambda_{22}=0,302 ; \lambda_{40}=0,107 ; \lambda_{55}=0,426$ \\
\hline 23 & $22,30,40$ y 61 & $\lambda_{22}=0,638 ; \lambda_{30}=0,267 ; \lambda_{40}=0,381 ; \lambda_{61}=0,040$ \\
\hline 29 & $8,16,28,54$ y 55 & $\lambda_{8}=0,163 ; \lambda_{16}=0,004 ; \lambda_{28}=0,872 ; \lambda_{54}=0,367 ; \lambda_{55}=0,392$ \\
\hline 31 & $8,16,22,30,40$ y 61 & $\lambda_{8}=0,83 ; \lambda_{16}=1,11 ; \lambda_{22}=0,01 ; \lambda_{30}=0,06 ; \lambda_{40}=1,47 ; \lambda_{61}=0,34$ \\
\hline 32 & $8,28,40$ y 54 & $\lambda_{8}=0,251 ; \lambda_{28}=0,130 ; \lambda_{40}=0,269 ; \lambda_{54}=0,2781$ \\
\hline 39 & 8,54 y 55 & $\lambda_{8}=1,204 ; \lambda_{54}=0,054 ; \lambda_{55}=0,367$ \\
\hline 53 & 8 y 30 & $\lambda_{8}=2,271 ; \lambda_{30}=0,350$ \\
\hline
\end{tabular}


EI DEA proporciona las unidades ineficientes, así como información acerca de los grupos de referencia de cada unidad, pero también los objetivos óptimos de producción y de consumo que los departamentos ineficientes deben alcanzar para ser catalogados como eficientes. Estos objetivos se calculan aplicando directamente el índice de eficiencia y las correspondientes variables de holgura a los valores reales de la unidad analizada. Los resultados, en términos porcentuales, aparecen recogidos en la tabla 8, para el caso de los departamentos experimentales y en la tabla 9 para los no experimentales.

Tabla 8: Reducción potencial en los inputs e incremento potencial de outputs. Departamentos experimentales ineficientes.

\begin{tabular}{|c|c|c|c|c|c|c|c|c|c|}
\hline Departamento & PDI & PD & PP & PED & $\mathrm{NC}$ & NA & NATC & NP & $\mathrm{IPI}$ \\
\hline Departamento 2 & $0 \%$ & $31 \%$ & $0 \%$ & $94 \%$ & $1 \%$ & $1 \%$ & $1437 \%$ & $79 \%$ & $334 \%$ \\
\hline Departamento 5 & $0 \%$ & $0 \%$ & $0 \%$ & $10 \%$ & $10 \%$ & $10 \%$ & $335 \%$ & $10 \%$ & $186 \%$ \\
\hline Departamento 6 & $0 \%$ & $0 \%$ & $0 \%$ & $69 \%$ & $14 \%$ & $14 \%$ & $8 \%$ & $14 \%$ & $349 \%$ \\
\hline Departamento 10 & $30 \%$ & $0 \%$ & $0 \%$ & $39 \%$ & $160 \%$ & $39 \%$ & $57 \%$ & $39 \%$ & \\
\hline Departamento 24 & $0 \%$ & $0 \%$ & $14 \%$ & $64 \%$ & $16 \%$ & $16 \%$ & & $20 \%$ & $16 \%$ \\
\hline Departamento 26 & $0 \%$ & $44 \%$ & $0 \%$ & $285 \%$ & $10 \%$ & $10 \%$ & $3 \%$ & $10 \%$ & $10 \%$ \\
\hline Departamento 33 & $0 \%$ & $9 \%$ & $0 \%$ & $110 \%$ & $11 \%$ & $11 \%$ & $152 \%$ & $11 \%$ & $347 \%$ \\
\hline Departamento 35 & $0 \%$ & $0 \%$ & $0 \%$ & $196 \%$ & $54 \%$ & $54 \%$ & $314 \%$ & $58 \%$ & $95 \%$ \\
\hline Departam & $3 \%$ & $0 \%$ & $0 \%$ & $143 \%$ & $2 \%$ & $9 \%$ & $6 \%$ & $2 \%$ & $33 \%$ \\
\hline Departam & $22 \%$ & $26 \%$ & $0 \%$ & $15 \%$ & $15 \%$ & $15 \%$ & $322 \%$ & $15 \%$ & $15 \%$ \\
\hline Departamento 47 & $0 \%$ & $64 \%$ & $20 \%$ & $57 \%$ & $64 \%$ & $535 \%$ & $41 \%$ & $41 \%$ & $382 \%$ \\
\hline Departam & $0 \%$ & $0 \%$ & $34 \%$ & $16 \%$ & $44 \%$ & $16 \%$ & $195 \%$ & $16 \%$ & $16 \%$ \\
\hline Departam & $0 \%$ & $0 \%$ & $2 \%$ & $36 \%$ & $36 \%$ & $169 \%$ & $265 \%$ & $36 \%$ & $36 \%$ \\
\hline Departamento 58 & $3 \%$ & $0 \%$ & $0 \%$ & $327 \%$ & $27 \%$ & $100 \%$ & $410 \%$ & $27 \%$ & $88 \%$ \\
\hline Departamento 59 & $0 \%$ & $0 \%$ & $0 \%$ & $3 \%$ & $3 \%$ & $60 \%$ & $239 \%$ & $3 \%$ & $198 \%$ \\
\hline Media & $4 \%$ & $12 \%$ & $5 \%$ & $98 \%$ & $31 \%$ & $71 \%$ & $305 \%$ & $25 \%$ & $150 \%$ \\
\hline
\end{tabular}

Tabla 9: Reducción potencial en los inputs e incremento potencial de outputs. Departamentos no experimentales ineficientes.

\begin{tabular}{|l|c|c|c|c|c|c|c|c|c|}
\hline \multicolumn{1}{|c|}{ Departamento } & PDI & PD & PP & PED & NC & NA & NATC & NP & IPI \\
\hline \hline Departamento 1 & $5 \%$ & $0 \%$ & $0 \%$ & $21 \%$ & $21 \%$ & $21 \%$ & $225 \%$ & $21 \%$ & $30 \%$ \\
\hline Departamento 14 & $0 \%$ & $0 \%$ & $2 \%$ & $64 \%$ & $56 \%$ & $56 \%$ &.. & $56 \%$ &.. \\
\hline Departamento 15 & $0 \%$ & $0 \%$ & $0 \%$ & $62 \%$ & $18 \%$ & $18 \%$ & $150 \%$ & $24 \%$ &.. \\
\hline Departamento 19 & $0 \%$ & $0 \%$ & $16 \%$ & $98 \%$ & $135 \%$ & $16 \%$ & $16 \%$ & $16 \%$ & $138 \%$ \\
\hline Departamento 20 & $0 \%$ & $0 \%$ & $2 \%$ & $6 \%$ & $31 \%$ & $6 \%$ & $568 \%$ & 110 & $225 \%$ \\
\hline Departamento 21 & $0 \%$ & $21 \%$ & $0 \%$ & $45 \%$ & $30 \%$ & $11 \%$ & $11 \%$ & $11 \%$ &.. \\
\hline Departamento 23 & $0 \%$ & $8 \%$ & $0 \%$ & $76 \%$ & $5 \%$ & $5 \%$ & $84 \%$ & $5 \%$ & $13 \%$ \\
\hline Departamento 29 & $0 \%$ & $0 \%$ & $0 \%$ & $89 \%$ & $8 \%$ & $8 \%$ & $115 \%$ & $8 \%$ & $745 \%$ \\
\hline Departamento 31 & $0 \%$ & $0 \%$ & $0 \%$ & 196 & $36 \%$ & $36 \%$ & $36 \%$ & $36 \%$ & $127 \%$ \\
\hline Departamento 32 & $1 \%$ & $0 \%$ & $0 \%$ & $16 \%$ & $46 \%$ & $16 \%$ & $16 \%$ & $91 \%$ &.. \\
\hline Departamento 39 & $0 \%$ & $0 \%$ & $3 \%$ & $31 \%$ & $4 \%$ & $4 \%$ & $131 \%$ & 187 &.. \\
\hline Departamento 53 & $0 \%$ & $0 \%$ & $9 \%$ & $93 \%$ & $47 \%$ & $131 \%$ & $230 \%$ & $91 \%$ &.. \\
\hline & $1 \%$ & $2 \%$ & $3 \%$ & $66 \%$ & $36 \%$ & $27 \%$ & $144 \%$ & $55 \%$ & $213 \%$ \\
\hline
\end{tabular}

De la tabla 8 se puede destacar que, en media, los departamentos experimentales ineficientes deben incrementar el número de alumnos de tercer ciclo (NATC) en un 305\% y los ingresos de proyectos de investigación (IPI) en un 150\%. Asimismo, para ser eficientes, deben reducir el personal docente (PD) en un $12 \%$ e incrementar el porcentaje de profesores con buena valoración en la encuesta de evaluación (PED) en un 98\%. De la tabla 9 cabe destacar que, en media, los departamentos no experimentales ineficientes deben incrementar el número de alumnos de tercer 
ciclo (NATC) en un 144\% y los ingresos de proyectos de investigación (IPI) en un 213\%. Asimismo, para ser eficientes, deben incrementar el porcentaje de profesores con buena valoración en la encuesta de evaluación (PED) en un 66\%.

Los resultados muestran las mejoras potenciales que pueden realizar los departamentos universitarios para desarrollar su actividad de forma eficiente. No obstante, los objetivos de producción y consumo de los departamentos ineficientes deben interpretarse siempre con cautela. Debe ser el propio departamento, quien, a partir de los resultados mostrados, tome las decisiones oportunas. Hay que tener presente que cada departamento tiene sus particularidades, las cuales pueden facilitar o dificultar el acercamiento a determinados objetivos. Además, hay factores ajenos al control del propio departamento y que el modelo DEA ignora, que también pueden dificultar alcanzar los óptimos preestablecidos.

\section{CONCLUSIONES}

El interés del presente trabajo se ha centrado en el análisis del grado de eficiencia con el que se gestionan los recursos universitarios. En este sentido, se ha puesto de manifiesto que es posible medir la eficiencia con la que actúan los departamentos universitarios. El análisis realizado ofrece evidencia empírica de la posibilidad de evaluar la eficiencia con la que actúan los departamentos de la ULL.

En relación a los departamentos ineficientes se puede acentuar la necesidad de incrementar los resultados obtenidos en algunas de las variables utilizadas para el análisis. Del mismo modo se ha obtenido información adicional, relacionada con los grupos de referencia y con los objetivos a alcanzar por cada departamento, lo que permite tener un mayor conocimiento de la actividad departamental.

Los resultados mostrados pueden facilitar a los departamentos la toma de decisiones y la planificación de sus actividades. El conocimiento de la posición de un departamento con respecto al resto ayuda a conocer sus puntos débiles y fuertes y, por tanto, a tomar las medidas correctoras necesarias o a potenciar sus fortalezas.

En este sentido, la utilidad del DEA para la gestión de los departamentos radica en que proporciona puntos operativos de referencia que sugieren líneas de acción para una mejor asignación de recursos que pueden ponerse en práctica en aquellos departamentos que se han revelado menos eficientes.

En resumen, los departamentos, como centros de gestión, deben contar con información sobre su modo de actuar, con el fin de poder realizar su propia planificación de objetivos y la programación de los medios necesarios para su consecución. A través del análisis realizado se facilita dicha información.

\section{REFERENCIAS}

Álvarez Pinilla, A. (coord.); La medición de la eficiencia y la productividad, Ed. Pirámide, Madrid (2001).

Avkiran, N.K.; Investigating technical and scale efficiencies of Australian Universities through data envelopment analysis, Socio-Economic Planning Sciences: 35, 57-80 (2001).

Banker, R.D., A. Charnes y W. Cooper; Some models for estimating technical and scale inefficiencies in data envelopment analysis, Management Science: 30, 1078-1092 (1984).

Caballero, R., T. Galache, T. Gómez y A. Torrico; Análisis de la eficiencia vía DEA y multiobjetivo. Una aplicación al caso de la Universidad de Málaga, IX Jornadas AEDE, 81-96, Jaén, España, 21 y 22 de septiembre (2000). 
Caballero, R., y otros cuatro autores; A., Budgetary allocations and efficiency in the human resources policy of a university following multiple criteria, Economics of Education Review: 23, 6774 (2004).

Calderón Patier, C., D. Barruso Castillo y N. Rueda López; La medición de la eficiencia de las políticas públicas de I+D en España: una aplicación del Análisis Envolvente de Datos (DEA), XII Encuentro de Economía Pública, http://www.uib.es/congres/ecopub/, Palma de Mallorca, España, 3 y 4 de febrero (2005).

Charnes, A., W. Cooper, A. Lewin y L. Seiford; DEA: Theory, Methodology and Applications, Kluwer Academic Publishers, USA (1994).

Charnes, A.; W. Cooper y E. Rhodes; Short communication: Measuring efficiency of decision making units, European Journal of Operational Research: 3, 339 (1979).

Charnes, A., W. Cooper y E. Rhodes; Measuring Efficiency of Decision Making Units, European Journal of Operational Research: 2, 429-444 (1978).

Coelli, T., D.S. Prasada y G.E. Battese; An introduction efficiency and productivity analysis, Kluwer Academic Publishers, USA (1999).

Farrell, M.J.; The measurement of productive efficiency, Journal of the Royal Statistical Society: 120, 253-290 (1957).

Ganley, J.A. y J.S. Cubbin; Public Sector Efficiency Measurement Applications of Data Envelopment Analysis, Elsevier Science Publisher, Amsterdam (1992).

García Valderrama, T.; La medida y el control de la eficiencia en las instituciones universitarias, Sindicatura de Comptes, Valencia (1996).

Giménez García, V. y J.L. Martínez Parra; Eficiencia en los costes en la universidad: una aplicación a los departamentos de la UAB, X Jornadas AEDE, 461-472, Murcia, España, 28 y 29 de septiembre (2001).

Johnes, J.; Measuring teaching efficiency in higher education: An application of data envelopment analysis to economics graduates from UK Universities 1993, European Journal of Operational Research: 174, 443-456 (2006).

Lovell, C.A.K. y M.A. Muñiz Pérez; Eficiencia y productividad en el sector público, Papeles de Economía Española: 95, 47-65 (2003).

Martín Rivero, R.; La eficiencia productiva en el ámbito universitario: aspectos claves para su evaluación, Estudios de Economía Aplicada: 25(3), 793-812 (2007)

Martínez Cabrera, M.; La medición de la eficiencia en las instituciones de educación superior, Fundación BBVA, Bilbao (2003).

Nunamaker, T.T.; Using Data envelopment analysis to measure the efficiency on non-profit organisations: a critical evaluation, Managerial and Decision Economics: 6 (1), 50-58 (1985).

Sinuany-Stern, Z., A. Mehrez y A. Barboy; Academic departments efficiency via DEA, Computers \& Operations Research: 21(5), 543-556 (1994).

Yetano Sánchez de Muniaín, A.; Hacia la gestión estratégica en la administración local: el balanced scorecard, Colección: Estudios de Hacienda Publica, Instituto de Estudios Fiscales, Madrid (2007). 